\title{
The late stage of autophagy: cellular events and molecular regulation
}

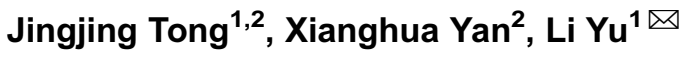 \\ ${ }^{1}$ State Key Laboratory of Biomembrane and Membrane Biotechnology, School of Life Science, Tsinghua University, Beijing \\ 100084, China \\ 2 College of Animal Sciences and Technology, Huazhong Agricultural University, Wuhan 430070, China \\ $\bowtie$ Correspondence: liyulab@mail.tsinghua.edu.cn \\ Received October 6, 2010 Accepted October 18, 2010
}

\begin{abstract}
Autophagy is an intracellular degradation system that delivers cytoplasmic contents to the lysosome for degradation. It is a "self-eating" process and plays a "house-cleaner" role in cells. The complex process consists of several sequential steps-induction, autophagosome formation, fusion of lysosome and autophagosome, degradation, efflux transportation of degradation products, and autophagic lysosome reformation. In this review, the cellular and molecular regulations of late stage of autophagy, including cellular events after fusion step, are summarized.
\end{abstract}

KEYWORDS autophagy, autophagosome, lysosome, fusion, degradation

\section{INTRODUCTION}

The balance between synthesis and degradation of cellular components is important for cellular homeostasis. In eukaryote cell, two powerful hydrolytic mechanisms, the proteasome system and the lysosome system, are responsible for degradation. Autophagy is a lysosome based degradation pathway. In contrast to the ubiquitin-proteasome system, which only degrades ubiquitinated proteins, autophagy can engulf and degrade large portion of cytoplasm in a highly regulated manner. There are three types of autophagy, macroautophagy, microautophagy, and chaperone-mediated autophagy (Klionsky, 2007). In this review we will focus on macroautophagy, hereafter referred to as autophagy. Based on the recent studies, the whole process of autophagy proceeds through the following sequential steps: 1 . induction of autophagy, 2. formation of autophagosome precursor, 3. formation of autophagosome, 4. fusion between autophagosome and lysosome, 5 . degradation of the contents, 6 . release of the degradation products to the cytoplasm, 7 . reformation of lysosome.

In eukaryotic cells, basal level autophagy occurs in most cell types in nutrient rich condition for its housekeeping functions (Mizushima, 2007). Dramatic upregulation of autophagy occurs in the presence of various stresses, such as starvation. mTOR (mammalian target of rapamycin) is one of the key regulators of cell growth. When nutrients are limited, mTOR is inactivated, which in turn induces autophagy. Autophagy is initiated by the formation or elongation of isolated membrane (IM). In yeast, the pre-autophagosomal structure (PAS) serves as the initial site of autophagy-related (Atg) protein recruitment. PAS is not observed in mammalian cells, but recent research found a structure named omegosome on endoplasmic reticulum. These structures provide a membrane platform for accumulation of autophagosomal proteins, expansion of autophagosomal membranes, and eventually formation of autophagosome (Yorimitsu and Klionsky, 2005; Mizushima, 2007; Xie and Klionsky, 2007; Nakatogawa et al., 2009; Hamasaki and Yoshimori, 2010). The origin of autophagosome membranes still remains controversial, but in mammalian cells, the endoplasmic reticulum seems to be the major source of autophagosome membrane (Axe et al., 2008). Autophagosome formation is regulated by a complicated molecular machinery. Among them, two ubiquitin-like conjugation systems, Atg12-Atg5 conjugation system and Atg8-PE conjugation system, participate in the expansion of autophagosome. After autophagosome formation, autophagosome will fuse with endosome and lysosome, to form a hybrid organelle named 
autolysosome. Inside autolysosome, cellular content engulfed by autophagosome is degraded. The degraded products such as amino acids and monosaccharide are released from autolysosome through lysosomal efflux transporter. Finally, lysosome components in autolysosome are recycled through an evolutionary conserved mechanism named autophagic lysosome reformation, to form the new functional lysosome.

So far, the formation of autophagosome has been subjected to extensive study and many excellent reviews have been dedicated to this topic (Longatti and Tooze, 2009; Ravikumar et al., 2009). The goal of this review is to provide an overview of recent advances in our understanding of cellular events after autophagosome formation. For the sake of clarity, this review divides the continuous cellular events following autolysosome formation into three parts: fusion between autophagosome and lysosome, degradation in autolysosome and efflux of degradation products, and finally, autophagic lysosome reformation.

\section{FUSION BETWEEN AUTOPHAGOSOMES AND LYSOSOMES}

After autophagosome formation, autophagosomes will fuse with lysosomes or vacuoles to form autolysosomes. The fusion process can be viewed as three steps: movement toward the target compartment, tethering/docking with the acceptor membrane, and the eventual fusion of the lipid bilayers. Fusion events between autophagosomes and lysosomes have been studied extensively. Studies indicate that the fusion between autophagosome and lysosomes in mammalian cells and yeast is a multi-step process regulated through complex molecular machinery.

\section{ESCRT}

The endosomal sorting complex required for transport (ESCRT) originally identified for their roles in sorting ubiquitinylated membrane proteins into multivesicular bodies has been found to play an important role in autophagosomelysosome fusion (Rothman and Wieland, 1996; Rusten et al., 2007; Raiborg and Stenmark, 2009). Under the condition of ESCRT-III inactivation, such as inhibiting the expression of ESCRT subunits through RNA interference or genetic mutation, autophagosome accumulated (Lee et al., 2007). Hrs belongs to ESCRT-0 complex and is a master regulator in endosomal protein sorting (Bache et al., 2002; Lloyd et al., 2002; Kanazawa et al., 2003). It contains a FYVE domain, and is localized to the autophagosomes, and its depletion significantly decreases the number of autolysosomes in cells (Tamai et al., 2007). These results indicate ESCRT-0 and ESCRT-III complex may be crucial in the autophagosomelysosome fusion. Also, there are data suggesting that ESCRT has a role in autophagosome formation (Rusten and
Stenmark, 2009). Further study on this mechanism will be needed to sort out the precise role of ESCRT in autophagy.

\section{Microtubules}

Autophagosomes appear to form randomly inside the mammalian cells. However, in most of cell types, lysosomes are clustered around the microtubule-organizing center (MTOC; located near the nucleus) (Jahreiss et al., 2008), thus fusion event requires lysosome or autophagosome to move closer. Recent literatures show that autophagosomes move along microtubules toward lysosomes in a dynein-dependent manner (Ravikumar et al., 2005). In perinuclear region, lysosomes and autophagosomes tether, dock and fuse with each other. But the mechanism for this directed movement is not well understood yet. Treating cells with microtubule disrupting reagents such as nocodazole and vinblastine causes delay in autophagosome-lysosome fusion (Aplin et al., 1992; Seglen et al., 1996). Conversely, microtubule stabilization by taxol increases the fusion between autophagic vacuoles and lysosomed. In addition, Fass et al. (2006), using time-lapse video microscopy, revealed only mature autophagosome but not isolation membrane can associate with microtubules and move along microtubule tracks. Recent research demonstrated autophagosomes move bi-directionally along microtubules. However, what drives autophagosomes moving along microtubules? Dynein, which moves toward the minus end of microtubules, is a motor protein (Gill et al., 1991; Schroer and Sheetz, 1991), and its partner dynactin, which is essential for motor activity, can interact with dynein to form a large complex. Modulating dynein by various methods, including using dynein ATPase adenosine deaminase inhibitor, RNAi or microinjecting anti-dynein intermediate chain antibodies, causes impairment of autophagosomes trafficking and decreases the fusion of these structures, suggesting dynein may be the motor protein that drives autophagosome along microtubules. Interestingly, Kimura et al. (2008) reported the average velocity of rapid autophagosome movement $(5 \mathrm{~m} / \mathrm{sec})$, which is consistent with the velocity of dynein motor $(4 \mathrm{~m} / \mathrm{sec})$ (Lakadamyali et al., 2003). So far, it is unknown how dynein interacts with autophagosome. One possibility is that dynein is directly or indirectly associated with LC3. Kimura et al. (2008) demonstrated GFP-LC3 and dynein partially co-localize in autophagosome, and anti-LC3 antibody microinjection inhibits autophagosome movement. Structural study has shown that the N-terminal extension in LC3 consists of two-alpha-helices (Sugawara et al., 2004; Kouno et al., 2005) and it binds to microtubules (Mann and Hammarback, 1994; Kouno et al., 2005). Microinjection of anti-N-terminal LC3 antibody abolishes autophagosome movement (Kimura et al., 2008). These data suggest the essential role of LC3 in autophagosome movement is possibly by promoting autophagosome connecting to microtubules. In yeast, treating cells with 
nocodazole does not affect autophagy, demonstrating that microtubules are not required for autophagy in yeast.

A novel protein FYCO1 (FYVE and coiled-coil [CC] domain containing 1) have been identified by Johansen group recently (Pankiv et al., 2010). It can associate with LC3 and Rab7 to form an adaptor complex, which promotes autophagic vesicles transportation along microtubule plus end. FYCO1 contains an N-terminal RUN domain, 850-aa-long CC region, FYVE domain, LIR (LC3-interacting region) motif, and globular domain. RUN domain is an a-helical proteinprotein interaction domain shown to bind to the small GTPases of Rab and Rap families (Callebaut et al., 2001; Recacha et al., 2009); CC region is responsible for the dimerization of $\mathrm{FYCO} 1$ and has many protein binding regions, C-terminal part of the $\mathrm{CC}$ is responsible for the co-localization of FYCO1 with Rab7, FYVE is a PI3P binding domain which may take part in the membrane targeting. They have also demonstrated that the $\mathrm{CC}$ domain in $\mathrm{FYCO} 1$ which is required for FYCO1 self-interacting is essential for membrane recruitment of FYCO1. LIR is another important domain in FYCO1. It is necessary and sufficient for mediating FYCO1 binding to LC3, and deletion of LIR resulted in loss of co-localization between FYCO1 and LC3. Endogenous FYCO1 is localized to punctuated structures concentrated in the juxtanuclear region, but FYCO1 redistributes to other parts of the cytoplasm during starvation. Depletion of FYCO1 leads to the accumulation of perinuclear clustering of autophagosomes, these autophagosomes co-localize with Rab7 but not the lysotracker. All these data suggested that FYCO1 binds to LC3 and Rab7 to form a complex which can promote autophagosome from perinuclear to MT plus end and regulate bidirectional transport of autophagosomes along the MT track (Pankiv et al., 2010).

\section{RAB, HOPS and SNARE}

So far, the evidence suggests that fusion between autophagosome and lysosome depends on the canonical fusion machinery, the RAB-SNARE (soluble N-ethylmalemide-sensitive factor attachment protein receptor) system, and the set of involved molecules are thought to be almost identical to those involved in vacuole-vacuole homotypic fusion (Cai et al., 2007). SNAREs are membrane-anchored proteins. They can adjoin their transmembrane domain through a conserved coiled-coil domain, which is required for regulated fusion between lipid bilayers (Ungermann and Langosch, 2005; Jahn and Scheller, 2006; Cai et al., 2007; Langosch et al., 2007). Based on their localization, SNAREs are classified as vesicle (v)-SNAREs (Ykt6/Nyv1) and target membrane(t)SNAREs (Vam3/Vam7/Vti1) (Rothman, 1994; Darsow et al., 1997; Nichols et al., 1997; Sato et al., 1998; Ungermann and Wickner, 1998; Ungermann et al., 1998, 1999). The fusioncompetent assembly of SNAREs localize on opposing membranes, termed the trans-SNARE complex (Weber et al., 1998). In mammalian cells, knocking down (t)-SNARE $\mathrm{Vti1} \mathrm{b}$ delays the maturation of autophagosomes (Parlati et al., 2000; Atlashkin et al., 2003). In yeast, it has been demonstrated that Vam3 and Vti1 are needed for the fusion between autophagosomes and vacuoles (Wang and Klionsky, 2003; Klionsky, 2005).

HOPS (homotypic fusion and protein sorting), is a conserved protein complex consisting of four $\mathrm{C}$-Vps proteins (Vps11, 16, 18, 33), and Vps41 and Vps39 (Price et al., 2000; Seals et al., 2000; Wurmser et al., 2000). HOPS interacts with t-SNARE vam3 and the vacuolar Rab GTPase Ypt7 through Vps33 (Sato et al., 2000; Dulubova et al., 2001; Stroupe et al., 2006). During fusion, the larger size of HOPS complex helps it reach over relatively longer distances compared to SNARE complex, thus, mediating the first contact between vacuoles (Cai et al., 2007). Recent research demonstrates HOPS complex prevents the disassembly of trans-SNARE complexes by Sec17/Sec18 during membrane fusion (Mima et al., 2008; Xu et al., 2010). Many data indicate HOPS complex plays a role in the early stages of docking at the vesicle surface and takes part in the vesicle fusion with SNAREs and Rab (Sato et al., 2000; Seals et al., 2000; Wurmser et al., 2000). Early in 1997, Stephanie E. Rieder group have demonstrated that Vps18 takes part in the delivery of autophagosome to the vacuole in yeast. The mutant of Vps18 shows numbers of autophagic bodies within the cytoplasm but not the vacuoles (Rieder and Emr, 1997). In Drosophila, the Vps18 homolog Deep orange (Dor) has previously been shown to mediate fusion of autophagosomes with lysosomes. It has been demonstrated class C Vps proteins are components of a heter-oligomeric protein complex that achieves their function (Rieder and Emr., 1997). Vps16 binds to Dor and Vps33 in Drosophila, and Vps16 knockdown causes loss of Dor and accumulation of autophagosomes (Pulipparacharuvil et al., 2005). Vam3p, a syntaxin homolog, is required for the autophagosomelysosome fusion. The mutant of Vam3 accumulated multiple autophagosomes in the cytoplasm but no detectable accumulation of autophagic bodies in the vacuole (Darsow et al., 1997). It is likely Vam3 and Vps33 do in fact function together to direct the docking and fusion of transport intermediates with the vacuole.

It is well established that Rab proteins which peripherally associate with membranes via a geranylgeranyl lipid tail play an important role in tethering/docking of vesicles to their target compartment during vesicles fusion. The vesicle associated GTP-bound form of Rab proteins is thought to be active and interact with its effector proteins. The GDPbound form of Rab proteins is inactive and disassociates from vesicles (Somsel Rodman and Wandinger-Ness, 2000). Each transport step requires activated Rab proteins binding to soluble factors which act as effector proteins (Marino and Heidi., 2001). HOPS complex seems to be the effector that mediates Rab7-dependent tethering (Price et al., 2000b). In 
yeast, Vps39 has been shown to confer GTPases exchange factor (GEF) activity to Ypt7p (the yeast Rab7 ortholog). In mammalian cells, hVps39 regulates the recruitment/activation of Rab7 onto the Rab5-labeled early endosomes. Rab effectors are not randomly distributed on the organelle membrane but are clustered in distinct functional domains (Novick and Zerial., 1997). The interaction between Rab effector and SNARE provides a new understanding how Rab proteins directly regulate SNARE function during fusion (Wurmser et al., 2000). Rab GTPases and their effectors provide the complementary specificity to SNARE complexes during membrane tethering and fusion. Based on the recent research, Rab7 GTPases is targeted to the autophagosome membrane and required for autophagosome maturation (Gutierrez et al., 2004; Jäger et al., 2004). Liang et al. (2008a) demonstrate that UVRAG bind to class C-Vps complex and this interaction promotes activation of Rab7 GTPase, which takes part in the mature of autophagosome (Liang et al., 2008b). Overexpression of a Rab7 dominant negative mutant impairs fusion between autophagosomes and the late endosome/lysosome (Gutierrez et al., 2004; Jäger et al., 2004). In addition, Rab24 shows a perinuclear reticular localization and partially overlapps with ER, cisGolgi, and the ER-Golgi intermediates compartment under normal condition. But its distribution changes dramatically under starvation. Co-localization between Rab24 and LC3 has been detected under starvation condition (Munafó and Colombo, 2002). Yoshimori group (2005) demonstrated that Rab24 may be involved in transportation of autophagosome membrane compartment to lysosome (Egami et al., 2005). Biochemical studies in hepatocytes have indicated that autophagosomes can fuse with endosomes to form amphisome before they fuse with lysosome. Such phenomenon can also be found in pancreatic cells, fibroblasts and HeLa cells. Rab11 is required for MVBs formation and its normal function (Satoh et al., 2005). Overexpression of wild-type Rab11 and its active mutant Rab11Q70L generates large MVBs marked by Rab11 and a remarkable colocalization with LC3. Mutant form of Rab11 hampered the interaction between MVBs and autophagosomes. And this process does not require Rab7 which plays a role in fusion between autophagosome and lysosome and amphisome and lysosome (Fader et al., 2008). Rab22 associates with early endosome and late endosome but not lysosome, and can affect the morphology and physiology of the endocytic pathway; interestingly, its active mutant can associate to lysosome and autophagosome (Mesa et al., 2001). Further research demonstrated it may play a role in autophagic process, but it will need more clear evidence.

\section{Others}

The peripheral membrane protein complex of Mon1-Ccz1 has been discussed as an additional factor involved in docking at the vacuole (Wang et al., 2002). Ccz1, initially discovered as a protein functionally linked to Ypt7 in yeast, is conserved in humans and C.elegans (Kucharczyk et al., 2001). Mon1 and Ccz1 are membrane-associated proteins, and Mon1-Ccz1 complex has been proposed to contain longin domains, which also have been detected in several other trafficking proteins such as SNAREs (Kucharczyk et al., 2000; Wang et al., 2002). In addition, either Mon 1 or Ccz1 requires the class $C$ subunits of the HOPS complex to associate with membrane. Wang et al. (2003) have demonstrated that vacuole fusion is strongly impaired when either protein is missing or when the proteins are inhibited by specific antibodies (Wang et al., 2003). It is possible that the Mon1-Ccz1 is a cofactor of the HOPS tethering complex in the fusion between autophagosome and lysosome (Wang and Klionsky, 2003; Klionsky et al., 2005).

LAMPs (lysosomal-associated membrane proteins) are heavily glycosylated lysosomal transmembrane proteins (Eskelinen et al., 2003). Lysosome membrane protein Lamp2 has been recently demonstrated a role in autophagosome maturation (Huynh et al., 2007; Saftig et al., 2008). Starvation-induced degradation of long-lived proteins is impaired in the lamp2-deficient hepatocytes (Tanaka et al., 2000). Interestingly, unlike Lamp2 single-deficient hepatocytes, the degradation of long-lived proteins is not affected in Lamp1/Lamp2 double deficient fibroblasts (Eskelinen et al., 2004), the difference is possibly due to the different autophagy rates in different cell types (Eskelinen, 2005). More interestingly, the delivery of Rab7 to autophagic vacuoles is impaired in the lamp double-deficient fibroblasts (Eskelinen et al., 2004). So there is a hypothesis that the role of Lamp2 in autophagosome maturation is to regulate the distribution or targeting of Rab7.

UVRAG (UV irradiation resistance-associated gene) is a Beclin1-binding autophagic tumor suppressor, and it has been reported to bind Beclin1 to take part in the formation of autophagosomes (Liang et al., 2006, 2007; Takahashi et al., 2007, 2008). Interestingly, Liang et al. (2008a) reported UVRAG can also interact with class C-VPS complex through Vps16 and has a role in the fusion between autophagosome and lysosome. And the role of UVRAG-Class C-Vps complex in autophagosome maturation is different from the role of UVRAG-Beclin1-mediated autophagosome formation. UVRAG mutant that was defective in binding to Beclin1 but can still interact with $\mathrm{C}-\mathrm{Vps}$ was able to regulate assembly of $\mathrm{LC}^{+}$and LAMP1 ${ }^{+}$structure. However, a mutant that cannot bind to C-Vps but still can bind to Beclin1 showed markedly attenuated ability to promote autophagosome maturation (Liang et al., 2008a). And this is due to UVRAG-Class C-Vps complex can activate Rab7 GTPase activity but not the UVRAG mutant, which cannot bind to Class C-Vps complex (Liang et al., 2008b; Peplowska et al., 2008).

AAA ATPases (AAA adenosine triphosphatases) was first defined as a subset of P-loop ATPases in the early 1990s, 
based on the homology within the ATP binding domain. They are conserved from prokaryotes to humans, and they use the energy by ATP hydrolysis to remodel their target substrates. They have many functions, such as vesicle transport, organelle assembly, and protein unfolding. Disassembly of protein complex by AAA proteins plays an important role in several cell biologic function including the fusion in autophagic pathway (White and Lauring, 2007; Mehrpour et al., 2010). NSF is one of the AAA proteins and it binds to SNARE complex and utilizes ATP hydrolysis to disassemble them in order to regenerate free SNAREs, thus allowing fusion proceed as we described above (Block et al., 1998; Ungermann et al., 1998). SKD1 (a mammalian homolog of yeast VPS4), another AAA protein, is critical for the disassembly of the ESCRT-III complex once cargo selection is completed. Overexpression of a SKD1 mutant which cannot hydrolyse ATP induced a defection in autophagosomes maturation (Shirahama et al., 1997; Nara et al., 2002; Rusten et al., 2007).

DRAM (damage-regulated autophagy modulator), a transmembrane protein present in the lysosome, has also been demonstrate a role in the later stage of autophagy in mammalian (Crighton et al., 2006).

\section{DEGRADATION AND LYSOSOME EFFLUX}

After autophagosome-lysosome fusion, the outer membrane of autophagosome is incorporated into lysosome/vacuole. The step of degradation may contain two steps: breakdown the autophagosome membrane to deliver its contents to the lysosome lumen, and the degradation of these contents by the various enzymes inside lysosome/vacuole.

Autophagosome is double membrane vesicle. After fusion with lysosome or vacuole, autophagosome membrane will be subject to lysosome degradation (Nakamura et al., 1997; Takeshige et al., 1992). Aut5p (a yeast homolog of mammalian Atg15), contains one to three potential transmembrane domains and a lipase active-site motif. It localizes on ER and is transported into vacuolar/lysosome via an autophagy-independent route (Odorizzi et al., 1998, 2000). As a lipase, it can disintegrate the membrane of autophagosome and release the contents into vacuole for degradation in Aut $5 \mathrm{p}^{-1-}$ cells accumulated autophagosomes (Epple et al., $2001,2003)$. The maturation of proaminopeptidase, which is dependent on delivery to the vacuole by autophagy, is impaired in Aut $5 \mathrm{p}^{-1-}$ cells.

The breakdown process of autophagic content depends on proteases and acidification of vacuole lumen in yeast (Takeshige et al., 1992; Nakamura et al., 1997). Proteinase A belongs to aspartic proteinase superfamily, which are proteolytic enzymogen. It is initially synthesized as an inactive precursor (zymogen), which transits to the endoplasmic reticulum. Proteinase A activation can occur either through proteinase $B$ catalyzed cleavage which is a vacuolar serine protease or via autoactivation. Proteinase $A$ is required to process inactive precursors of various enzymes (Parr et al, 2007). Experiments on the effects of gene disruption and inhibitors of proteases (proteinase A and B) showed the accumulation of autophagic bodies in wild-type cells and that these autophagic bodies disappeared rapidly from the vacuoles once the proteases activity were restored (Mechler and Wolf, 1981; Takeshige et al., 1992; Klionsky, 2005).

The degradation products, such as amino acids and monosaccharide, will be transported out of lysosome/vacuole through a group of lysosomal transmembrane proteins named lysosomal efflux transporters (Lloyd, 1996). Atg22 was first identified as Aut 4 as an integral membrane protein located on membrane of lysosome/vacuole (Teter et al., 2001). It was first identified as a protein required for autophagic degradation (Suriapranata et al., 2000), however, the follow up work by Klionsky's group demonstrated that Atg22 is not essential for autophagic cargo degradation. Instead, they found Atg22 is a tyrosine and leucine efflux transporter on vacuolar membrane. The Atg22 mutant that they examined is auxotrophic for leucine which could be rescued by addition of leucine and the mutant shows inhibition of protein synthesis under autophagy-inducing condition.

\section{AUTOPHAGIC LYSOSOME REFORMATION}

One of the key roles of autophagy is to recycle nutrients through degradation of unessential cellular content. As we have mentioned above, autophagosome formation is triggered by mTOR inactivation shortly after starvation. Interestingly, Yu et al. (2010) recently reported the amino acids releasing form autolysosome will reactivate mTOR. mTOR reactivation will trigger the disassembly of autolysosome, resulting in a tubular structure which mainly contains lysosomal membrane components extended from autolysosome. These tubular structures are highly dynamic, undergoing constantly fission of budding process by which small Lamp1 positive vesicles named "proto-lysosome" are formed. Proto-lysosomes are not acidic and do not have degradation capacity, but over a period of 2-3 h, proto-lysosomes mature into functional lysosome through acquiring lysosomal lumen proteins by a M6PR-dependent mechanism. This process is named as autophagic lysosome reformation (ALR). ALR is directly regulated by mTOR activities. When mTOR is inhibited, ALR is blocked, resulting in enlarged long-lasting autolysosome. So far, the molecular machinery regulating ALR is largely unknown. Rab7 is required to disassociate from autolysosome before ALR, and forced staying of Rab7 on autolysosome membrane by overexpressing constitutive active form of Rab7 blocks ALR (Yu et al., 2010).

\section{CONCLUSION}

So far, later stage of autophagy have received relatively less 
attention comparing to the earlier stage of autophagy, however, recently work had demonstrated that the fusion, degradation and lysosome reformation are regulated by a set of elaborated and complicated molecular machinery, and defecting in these later stage autophagy events has serious physiologic consequence. At this point, better understanding the cellular events and of molecular regulation of later stage autophagy is clearly needed, as many intriguing questions still remain unsolved, for example, how lysosome recognizes and selectively fuses with "mature" autophagosome but not isolation membrane? What is the molecular machinery to regulate the formation of reformation tubules? How components from lysosome are separated from autophagosome components during autophagic lysosome reformation? We are confident that with more researchers from different background attracted into this emerging research direction, these questions can be solved soon.

\section{ABBREVIATIONS}

AAA ATPases, AAA adenosine triphosphatases; ALR, autophagic lysosome reformation; Atg, autophagy-related; Dor, Deep orange; DRAM, damage-regulated autophagy modulator; ESCRT, endosomal sorting complex required for transport; FYCO1, FYVE and coiled-coil [CC] domain containing 1; GEF, GTPases exchange factor; HOPS, homotypic fusion and protein sorting; IM, isolated membrane; LAMPs, lysosomal-associated membrane proteins; LIR, LC3-interacting region; MTOC, microtubule-organizing center; SNARE, soluble Nethylmalemide-sensitive factor attachment protein receptor; mTOR, mammalian target of rapamycin; PAS, pre-autophagosomal structure; t-SNAREs, target membrane SNAREs; UVRAG, UV irradiation resistance-associated gene; v-SNAREs, vesicle SNAREs

\section{REFERENCES}

Aplin, A., Jasionowski, T., Tuttle, D.L., Lenk, S.E., and Dunn, W.A. Jr. (1992). Cytoskeletal elements are required for the formation and maturation of autophagic vacuoles. J Cell Physiol 152, 458-466.

Atlashkin, V., Kreykenbohm, V., Eskelinen, E.-L., Wenzel, D., Fayyazi, A., and Fischer von Mollard, G. (2003). Deletion of the SNARE vti1b in mice results in the loss of a single SNARE partner, syntaxin 8. Mol Cell Biol 23, 5198-5207.

Axe, E.L., Walker, S.A., Manifava, M., Chandra, P., Roderick, H.L., Habermann, A., Griffiths, G., and Ktistakis, N.T. (2008). Autophagosome formation from membrane compartments enriched in phosphatidylinositol 3-phosphate and dynamically connected to the endoplasmic reticulum. J Cell Biol 182, 685-701.

Bache, K.G., Raiborg, C., Mehlum, A., Madshus, I.H., and Stenmark, H. (2002). Phosphorylation of Hrs downstream of the epidermal growth factor receptor. Eur J Biochem 269, 3881-3887.

Block, M.R., Glick, B.S., Wilcox, C.A., Wieland, F.T., and Rothman, J. E. (1988). Purification of an N-ethylmaleimide-sensitive protein catalyzing vesicular transport. Proc Natl Acad Sci U S A 85, 7852-7856.

Cai, H., Reinisch, K., and Ferro-Novick, S. (2007). Coats, tethers, Rabs, and SNAREs work together to mediate the intracellular destination of a transport vesicle. Dev Cell 12, 671-682.
Callebaut, I., de Gunzburg, J., Goud, B., and Mornon, J.P. (2001). RUN domains: a new family of domains involved in Ras-like GTPase signaling. Trends Biochem Sci 26, 79-83.

Cao, X., and Barlowe, C. (2000). Asymmetric requirements for a Rab GTPase and SNARE proteins in fusion of COPII vesicles with acceptor membranes. J Cell Biol 149, 55-66.

Crighton, D., Wilkinson, S., O'Prey, J., Syed, N., Smith, P., Harrison, P.R., Gasco, M., Garrone, O., Crook, T., and Ryan, K.M. (2006). DRAM, a p53-induced modulator of autophagy, is critical for apoptosis. Cell 126, 121-134.

Darsow, T., Rieder, S.E., and Emr, S.D. (1997). A multispecificity syntaxin homologue, Vam3p, essential for autophagic and biosynthetic protein transport to the vacuole. J Cell Biol 138, 517-529.

Dulubova, I., Yamaguchi, T., Wang, Y., Südhof, T.C., and Rizo, J. (2001). Vam3p structure reveals conserved and divergent properties of syntaxins. Nat Struct Biol 8, 258-264.

Egami, Y., Kiryu-Seo, S., Yoshimori, T., and Kiyama, H. (2005). Induced expressions of Rab24 GTPase and LC3 in nerve-injured motor neurons. Biochem Biophys Res Commun 337, 1206-1213.

Epple, U.D., Suriapranata, I., Eskelinen, E.-L., and Thumm, M. (2001). Aut5/Cvt17p, a putative lipase essential for disintegration of autophagic bodies inside the vacuole. J Bacteriol 183, 5942-5955.

Epple, U.D., Eskelinen, E.L., and Thumm, M. (2003). Intravacuolar membrane lysis in Saccharomyces cerevisiae. Does vacuolar targeting of Cvt17/Aut5p affect its function? J Biol Chem 278, 7810-7821.

Eskelinen, E.L. (2005). Maturation of autophagic vacuoles in mammalian cells. Autophagy 1, 1-10.

Eskelinen, E.L., Tanaka, Y., and Saftig, P. (2003). At the acidic edge: emerging functions for lysosomal membrane proteins. Trends Cell Biol 13, 137-145.

Eskelinen, E.L., Schmidt, C.K., Neu, S., Willenborg, M., Fuertes, G., Salvador, N., Tanaka, Y., Lüllmann-Rauch, R., Hartmann, D., Heeren, J., et al. (2004). Disturbed cholesterol traffic but normal proteolytic function in LAMP-1/LAMP-2 double-deficient fibroblasts. Mol Biol Cell 15, 3132-3145.

Fader, C.M., Sánchez, D., Furlán, M., and Colombo, M.I. (2008). Induction of autophagy promotes fusion of multivesicular bodies with autophagic vacuoles in k562 cells. Traffic 9, 230-250.

Fass, E., Shvets, E., Degani, I., Hirschberg, K., and Elazar, Z. (2006). Microtubules support production of starvation-induced autophagosomes but not their targeting and fusion with lysosomes. J Biol Chem 281, 36303-36316.

Gasch, A.P., Spellman, P.T., Kao, C.M., Carmel-Harel, O., Eisen, M. B., Storz, G., Botstein, D., and Brown, P.O. (2000). Genomic expression programs in the response of yeast cells to environmental changes. Mol Biol Cell 11, 4241-4257.

Gill, S.R., Schroer, T.A., Szilak, I., Steuer, E.R., Sheetz, M.P., and Cleveland, D.W. (1991). Dynactin, a conserved, ubiquitously expressed component of an activator of vesicle motility mediated by cytoplasmic dynein. J Cell Biol 115, 1639-1650.

Gutierrez, M.G., Munafó, D.B., Berón, W., and Colombo, M.I. (2004). Rab7 is required for the normal progression of the autophagic pathway in mammalian cells. J Cell Sci 117, 2687-2697.

Hamasaki, M., and Yoshimori, T. (2010). Where do they come from? Insight into autophagosome formation. FEBS Lett 584, 1296-1301. 
Hayakawa, A., Hayes, S.J., Lawe, D.C., Sudharshan, E., Tuft, R., Fogarty, K., Lambright, D., and Corvera, S. (2004). Structural basis for endosomal targeting by FYVE domains. J Biol Chem 279, 5958-5966.

Huynh, K.K., Eskelinen, E.L., Scott, C.C., Malevanets, A., Saftig, P., and Grinstein, S. (2007). LAMP proteins are required for fusion of lysosomes with phagosomes. EMBO J 26, 313-324.

Itoh, T., Fujita, N., Kanno, E., Yamamoto, A., Yoshimori, T., and Fukuda, M. (2008). Golgi-resident small GTPase Rab33B interacts with Atg16L and modulates autophagosome formation. Mol Biol Cell 19, 2916-2925.

Jäger, S., Bucci, C., Tanida, I., Ueno, T., Kominami, E., Saftig, P., and Eskelinen, E.L. (2004). Role for Rab7 in maturation of late autophagic vacuoles. J Cell Sci 117, 4837-4848.

Jahn, R., and Scheller, R.H. (2006). SNAREs-engines for membrane fusion. Nat Rev Mol Cell Biol 7, 631-643.

Jahreiss, L., Menzies, F.M., and Rubinsztein, D.C. (2008). The itinerary of autophagosomes: from peripheral formation to kissand-run fusion with lysosomes. Traffic 9, 574-587.

Kanazawa, C., Morita, E., Yamada, M., Ishii, N., Miura, S., Asao, H., Yoshimori, T., and Sugamura, K. (2003). Effects of deficiencies of STAMs and Hrs, mammalian class $E$ Vps proteins, on receptor downregulation. Biochem Biophys Res Commun 309, 848-856.

Kimura, S., Noda, T., and Yoshimori, T. (2008). Dynein-dependent movement of autophagosomes mediates efficient encounters with lysosomes. Cell Struct Funct 33, 109-122.

Klionsky, D.J. (2005). The molecular machinery of autophagy: unanswered questions. J Cell Sci 118, 7-18.

Klionsky, D.J. (2007). Autophagy: from phenomenology to molecular understanding in less than a decade. Nat Rev Mol Cell Biol 8, 931-937.

Kouno, T., Mizuguchi, M., Tanida, I., Ueno, T., Kanematsu, T., Mori, Y., Shinoda, H., Hirata, M., Kominami, E., and Kawano, K. (2005). Solution structure of microtubule-associated protein light chain 3 and identification of its functional subdomains. J Biol Chem 280, 24610-24617.

Kucharczyk, R., Dupre, S., Avaro, S., Haguenauer-Tsapis, R., Słonimski, P.P., and Rytka, J. (2000). The novel protein Ccz1p required for vacuolar assembly in Saccharomyces cerevisiae functions in the same transport pathway as Ypt7p. J Cell Sci 113, 4301-4311.

Kucharczyk, R., Kierzek, A.M., Slonimski, P.P., and Rytka, J. (2001). The Ccz1 protein interacts with Ypt7 GTPase during fusion of multiple transport intermediates with the vacuole in S. cerevisiae. J Cell Sci 114, 3137-3145.

Kutateladze, T.G. (2006). Phosphatidylinositol 3-phosphate recognition and membrane docking by the FYVE domain. Biochim Biophys Acta 1761, 868-877.

Lakadamyali, M., Rust, M.J., Babcock, H.P., and Zhuang, X. (2003). Visualizing infection of individual influenza viruses. Proc Natl Acad Sci U S A 100, 9280-9285.

Langosch, D., Hofmann, M., and Ungermann, C. (2007). The role of transmembrane domains in membrane fusion. Cell Mol Life Sci 64, 850-864.

Lee, J.A., Beigneux, A., Ahmad, S.T., Young, S.G., and Gao, F.B. (2007). ESCRT-III dysfunction causes autophagosome accumulation and neurodegeneration. Curr Biol 17, 1561-1567.

Liang, C., Feng, P., Ku, B., Dotan, I., Canaani, D., Oh, B.H., and Jung,
J.U. (2006). Autophagic and tumour suppressor activity of a novel Beclin1-binding protein UVRAG. Nat Cell Biol 8, 688-699.

Liang, C., Feng, P., Ku, B., Oh, B.H., Jung, J.U., Oh, B., and Jung, J. (2007). UVRAG: a new player in autophagy and tumor cell growth. Autophagy 3, 69-71.

Liang, C., Lee, J.S., Inn, K.S., Gack, M.U., Li, Q., Roberts, E.A., Vergne, I., Deretic, V., Feng, P., Akazawa, C., et al. (2008a). Beclin1-binding UVRAG targets the class C Vps complex to coordinate autophagosome maturation and endocytic trafficking. Nat Cell Biol 10, 776-787.

Liang, C., Sir, D., Lee, S., Ou, J.H., and Jung, J.U. (2008b). Beyond autophagy: the role of UVRAG in membrane trafficking. Autophagy 4, 817-820.

Lindmo, K., Simonsen, A., Brech, A., Finley, K., Rusten, T.E., and Stenmark, H. (2006). A dual function for Deep orange in programmed autophagy in the Drosophila melanogaster fat body. Exp Cell Res 312, 2018-2027.

Lloyd, J.B. (1996). Metabolite efflux and influx across the lysosome membrane. Subcell Biochem 27, 361-386.

Lloyd, T.E., Atkinson, R., Wu, M.N., Zhou, Y., Pennetta, G., and Bellen, H.J. (2002). Hrs regulates endosome membrane invagination and tyrosine kinase receptor signaling in Drosophila. Cell 108, 261-269.

Longatti, A., and Tooze, S.A. (2009). Vesicular trafficking and autophagosome formation. Cell Death Differ 16, 956-965.

Lupas, A., Van Dyke, M., and Stock, J. (1991). Predicting coiled coils from protein sequences. Science 252, 1162-1164.

Mann, S.S., and Hammarback, J.A. (1994). Molecular characterization of light chain 3. A microtubule binding subunit of MAP1A and MAP1B. J Biol Chem 269, 11492-11497.

Marchler-Bauer, A., Anderson, J.B., Chitsaz, F., Derbyshire, M.K., DeWeese-Scott, C., Fong, J.H., Geer, L.Y., Geer, R.C., Gonzales, N.R., Gwadz, M., et al. (2009). CDD: specific functional annotation with the Conserved Domain Database. Nucleic Acids Res 37, D205-D210.

Marino, Z., and Heidi, M. (2001). Rab proteins as membrane prganizers. Natl Rev 2, 107-118.

Mechler, B., and Wolf, D.H. (1981). Analysis of proteinase A function in yeast. Eur J Biochem 121, 47-52.

Mehrpour, M., Esclatine, A., Beau, I., and Codogno, P. (2010). Overview of macroautophagy regulation in mammalian cells. Cell Res 20, 748-762.

Mesa, R., Salomón, C., Roggero, M., Stahl, P.D., and Mayorga, L.S. (2001). Rab22a affects the morphology and function of the endocytic pathway. J Cell Sci 114, 4041-4049.

Mima, J., Hickey, C.M., Xu, H., Jun, Y., and Wickner, W. (2008). Reconstituted membrane fusion requires regulatory lipids, SNAREs and synergistic SNARE chaperones. EMBO J 27, 2031-2042.

Mizushima, N. (2007). Autophagy: process and function. Genes Dev 21, 2861-2873.

Munafó, D.B., and Colombo, M.I. (2002). Induction of autophagy causes dramatic changes in the subcellular distribution of GFPRab24. Traffic 3, 472-482.

Nakamura, N., Matsuura, A., Wada, Y., and Ohsumi, Y. (1997). Acidification of vacuoles is required for autophagic degradation in the yeast, Saccharomyces cerevisiae. J Biochem 121, 338-344.

Nakatogawa, H., Suzuki, K., Kamada, Y., and Ohsumi, Y. (2009). 
Dynamics and diversity in autophagy mechanisms: lessons from yeast. Nat Rev Mol Cell Biol 10, 458-467.

Nara, A., Mizushima, N., Yamamoto, A., Kabeya, Y., Ohsumi, Y., and Yoshimori, T. (2002). SKD1 AAA ATPase-dependent endosomal transport is involved in autolysosome formation. Cell Struct Funct 27, 29-37.

Nichols, B.J., Ungermann, C., Pelham, H.R.B., Wickner, W.T., and Haas, A. (1997). Homotypic vacuolar fusion mediated by t- and vSNAREs. Nature 387, 199-202.

Novick, P., and Zerial, M. (1997). The diversity of Rab proteins in vesicle transport. Curr Opin Cell Biol 9, 496-504.

Odorizzi, G., Babst, M., and Emr, S.D. (1998). Fab1p Ptdlns(3)P 5kinase function essential for protein sorting in the multivesicular body. Cell 95, 847-858.

Odorizzi, G., Babst, M., and Emr, S.D. (2000). Phosphoinositide signaling and the regulation of membrane trafficking in yeast. Trends Biochem Sci 25, 229-235.

Olkkonen, V.M., Dupree, P., Killisch, I., Lütcke, A., Zerial, M., and Simons, K. (1993). Molecular cloning and subcellular localization of three GTP-binding proteins of the rab subfamily. J Cell Sci 106, 1249-1261.

Pankiv, S., Alemu, E.A., Brech, A., Bruun, J.A., Lamark, T., Overvatn, A., Bjørkøy, G., and Johansen, T. (2010). FYCO1 is a Rab7 effector that binds to LC3 and PI3P to mediate microtubule plus enddirected vesicle transport. J Cell Biol 188, 253-269.

Parlati, F., McNew, J.A., Fukuda, R., Miller, R., Söllner, T.H., and Rothman, J.E. (2000). Topological restriction of SNARE-dependent membrane fusion. Nature 407, 194-198.

Parr, C.L., Keates, R.A., Bryksa, B.C., Ogawa, M., and Yada, R.Y. (2007). The structure and function of Saccharomyces cerevisiae proteinase A. Yeast 24, 467-480.

Peplowska, K., Cabrera, M., and Ungermann, C. (2008). UVRAG reveals its second nature. Nat Cell Biol 10, 759-761.

Price, A., Seals, D., Wickner, W., and Ungermann, C. (2000a). The docking stage of yeast vacuole fusion requires the transfer of proteins from a cis-SNARE complex to a Rab/Ypt protein. J Cell Biol 148, 1231-1238.

Price, A., Wickner, W., and Ungermann, C. (2000b). Proteins needed for vesicle budding from the Golgi complex are also required for the docking step of homotypic vacuole fusion. J Cell Biol 148, 1223-1229.

Pulipparacharuvil, S., Akbar, M.A., Ray, S., Sevrioukov, E.A., Haberman, A.S., Rohrer, J., and Krämer, H. (2005). Drosophila Vps16A is required for trafficking to lysosomes and biogenesis of pigment granules. J Cell Sci 118, 3663-3673.

Raiborg, C., and Stenmark, H. (2009). The ESCRT machinery in endosomal sorting of ubiquitylated membrane proteins. Nature 458, 445-452.

Ravikumar, B., Acevedo-Arozena, A., Imarisio, S., Berger, Z., Vacher, C., O'Kane, C.J., Brown, S.D., and Rubinsztein, D.C. (2005). Dynein mutations impair autophagic clearance of aggregate-prone proteins. Nat Genet 37, 771-776.

Ravikumar, B., Futter, M., Jahreiss, L., Korolchuk, V.I., Lichtenberg , M., Luo, S., Massey, D.C., Menzies, F.M., Narayanan, U., Renna, M., et al. (2009). Mammalian macroautophagy at a glance. J Cell Biol 122, 1707-1711.

Recacha, R., Boulet, A., Jollivet, F., Monier, S., Houdusse, A., Goud, B., and Khan, A.R. (2009). Structural basis for recruitment of Rab6- interacting protein 1 to Golgi via a RUN domain. Structure 17, 21-30.

Rieder, S.E., and Emr, S.D. (1997). A novel RING finger protein complex essential for a late step in protein transport to the yeast vacuole. Mol Biol Cell 8, 2307-2327.

Rothman, J.E. (1994). Mechanisms of intracellular protein transport. Nature 372, 55-63.

Rothman, J.E., and Wieland, F.T. (1996). Protein sorting by transport vesicles. Science 272, 227-234.

Rusten, T.E., and Stenmark, H. (2009). How do ESCRT proteins control autophagy? J Cell Sci 122, 2179-2183.

Rusten, T.E., Vaccari, T., Lindmo, K., Rodahl, L.M., Nezis, I.P., SemJacobsen, C., Wendler, F., Vincent, J.P., Brech, A., Bilder, D., et al. (2007). ESCRTs and Fab1 regulate distinct steps of autophagy. Curr Biol 17, 1817-1825.

Saftig, P., Beertsen, W., and Eskelinen, E.L. (2008). LAMP-2: a control step for phagosome and autophagosome maturation. Autophagy 4, 510-512.

Sato, T.K., Darsow, T., and Emr, S.D. (1998). Vam7p, a SNAP-25-like molecule, and Vam3p, a syntaxin homolog, function together in yeast vacuolar protein trafficking. Mol Cell Biol 18, 5308-5319.

Sato, T.K., Rehling, P., Peterson, M.R., and Emr, S.D. (2000). Class C Vps protein complex regulates vacuolar SNARE pairing and is required for vesicle docking/fusion. Mol Cell 6, 661-671.

Satoh, A.K., O'Tousa, J.E., Ozaki, K., and Ready, D.F. (2005). Rab11 mediates post-Golgi trafficking of rhodopsin to the photosensitive apical membrane of Drosophila photoreceptors. Development 132, 1487-1497.

Schroer, T.A., and Sheetz, M.P. (1991). Two activators of microtubulebased vesicle transport. J Cell Biol 115, 1309-1318.

Seals, D.F., Eitzen, G., Margolis, N., Wickner, W.T., and Price, A. (2000). A Ypt/Rab effector complex containing the Sec1 homolog Vps33p is required for homotypic vacuole fusion. Proc Natl Acad Sci U S A 97, 9402-9407.

Seglen, P.O., Berg, T.O., Blankson, H., Fengsrud, M., Holen, I., and Strømhaug, P.E. (1996). Structural aspects of autophagy. Adv Exp Med Biol 389, 103-111.

Shirahama, K., Noda, T., and Ohsumi, Y. (1997). Mutational analysis of Csc1/Nps4p: involvement of endosome in regulation of autophagy in yeast. Cell Struct Funct 22, 501-509.

Söllner, T., Bennett, M.K., Whiteheart, S.W., Scheller, R.H., and Rothman, J.E. (1993). A protein assembly-disassembly pathway in vitro that may correspond to sequential steps of synaptic vesicle docking, activation, and fusion. Cell 75, 409-418.

Somsel Rodman, J., and Wandinger-Ness, A. (2000). Rab GTPases coordinate endocytosis. J Cell Sci 113, 183-192.

Stroupe, C., Collins, K.M., Fratti, R.A., and Wickner, W. (2006). Purification of active HOPS complex reveals its affinities for phosphoinositides and the SNARE Vam7p. EMBO J 25, 1579-1589.

Sugawara, K., Suzuki, N.N., Fujioka, Y., Mizushima, N., Ohsumi, Y., and Inagaki, F. (2004). The crystal structure of microtubuleassociated protein light chain 3 , a mammalian homologue of Saccharomyces cerevisiae Atg8. Genes Cells 9, 611-618.

Suriapranata, I., Epple, U.D., Bernreuther, D., Bredschneider, M., Sovarasteanu, K., and Thumm, M. (2000). The breakdown of autophagic vesicles inside the vacuole depends on Aut4p. J Cell Sci 113, 4025-4033. 
Takahashi, Y., Coppola, D., Matsushita, N., Cualing, H.D., Sun, M., Sato, Y., Liang, C., Jung, J.U., Cheng, J.Q., Mulé, J.J., et al. (2007). Bif-1 interacts with Beclin 1 through UVRAG and regulates autophagy and tumorigenesis. Nat Cell Biol 9, 1142-1151.

Takahashi, Y., Meyerkord, C.L., and Wang, H.G. (2008). BARgaining membranes for autophagosome formation: Regulation of autophagy and tumorigenesis by Bif-1/Endophilin B1. Autophagy 4, 121-124.

Takeshige, K., Baba, M., Tsuboi, S., Noda, T., and Ohsumi, Y. (1992). Autophagy in yeast demonstrated with proteinase-deficient mutants and conditions for its induction. J Cell Biol 119, 301-311.

Tamai, K., Tanaka, N., Nara, A., Yamamoto, A., Nakagawa, I., Yoshimori, T., Ueno, Y., Shimosegawa, T., and Sugamura, K. (2007). Role of Hrs in maturation of autophagosomes in mammalian cells. Biochem Biophys Res Commun 360, 721-727.

Tanaka, Y., Guhde, G., Suter, A., Eskelinen, E.L., Hartmann, D., Lüllmann-Rauch, R., Janssen, P.M., Blanz, J., von Figura, K., and Saftig, P. (2000). Accumulation of autophagic vacuoles and cardiomyopathy in LAMP-2-deficient mice. Nature 406, 902-906.

Teter, S.A., Eggerton, K.P., Scott, S.V., Kim, J., Fischer, A.M., and Klionsky, D.J. (2001). Degradation of lipid vesicles in the yeast vacuole requires function of Cvt17, a putative lipase. J Biol Chem 276, 2083-2087.

Ungermann, C., and Langosch, D. (2005). Functions of SNAREs in intracellular membrane fusion and lipid bilayer mixing. J Cell Sci 118, 3819-3828.

Ungermann, C., Nichols, B.J., Pelham, H.R.B., and Wickner, W. (1998). A vacuolar v-t-SNARE complex, the predominant form in vivo and on isolated vacuoles, is disassembled and activated for docking and fusion. J Cell Biol 140, 61-69.

Ungermann, C., von Mollard, G.F., Jensen, O.N., Margolis, N., Stevens, T.H., and Wickner, W. (1999). Three v-SNAREs and two tSNAREs, present in a pentameric cis-SNARE complex on isolated vacuoles, are essential for homotypic fusion. J Cell Biol 145, 1435-1442.

Ungermann, C., and Wickner, W. (1998). Vam7p, a vacuolar SNAP-
25 homolog, is required for SNARE complex integrity and vacuole docking and fusion. EMBO J 17, 3269-3276.

Wang, C.-W., and Klionsky, D.J. (2003). The molecular mechanism of autophagy. Mol Med 9, 65-76.

Wang, C.-W., Stromhaug, P.E., Kauffman, E.J., Weisman, L.S., and Klionsky, D.J. (2003). Yeast homotypic vacuole fusion requires the Ccz1-Mon1 complex during the tethering/docking stage. J Cell Biol 163, 973-985.

Wang, C.-W., Stromhaug, P.E., Shima, J., and Klionsky, D.J. (2002). The Ccz1-Mon1 protein complex is required for the late step of multiple vacuole delivery pathways. J Biol Chem 277, 47917-47927.

Weber, T., Zemelman, B.V., McNew, J.A., Westermann, B., Gmachl, M., Parlati, F., Söllner, T.H., and Rothman, J.E. (1998). SNAREpins: minimal machinery for membrane fusion. Cell 92, 759-772.

White, S.R., and Lauring, B. (2007). AAA + ATPases: achieving diversity of function with conserved machinery. Traffic 8 , 1657-1667.

Wurmser, A.E., Sato, T.K., and Emr S.D. (2000).New component of the vacuolar class $\mathrm{C}-\mathrm{Vps}$ complex couples nucleotide exchange on the Ypt7 GTPase to SNARE-dependent docking and fusion. J Cell Biol 151, 551-62.

Xie, Z., and Klionsky, D.J. (2007). Autophagosome formation: core machinery and adaptations. Nat Cell Biol 9, 1102-1109.

Xu, H., Jun, Y., Thompson, J., Yates, J., and Wickner, W. (2010). HOPS prevents the disassembly of trans-SNARE complexes by Sec17p/Sec1p during membrane fusion. J EMBO 29, 1948-1960.

Yang, Z., Huang, J., Geng, J., Nair, U., and Klionsky, D.J. (2006). Atg22 recycles amino acids to link the degradative and recycling functions of autophagy. Mol Biol Cell 17, 5094-5104.

Yorimitsu, T., and Klionsky, D.J. (2005). Autophagy: molecular machinery for self-eating. Cell Death Differ 12, 1542-1552.

Yu, L., McPhee, C.K., Zheng, L., Mardones, G.A., Rong, Y., Peng, J., Mi, N., Zhao, Y., Liu, Z., Wan, F., et al. (2010). Termination of autophagy and reformation of lysosomes regulated by mTOR. Nature 17, 942-946. 